\title{
EVALUACIÓN DE LA REFLECTIVIDAD DE HERBAZALES PANTANOSOS Y ÁREAS DEFORESTADAS UTILIZANDO IMÁGENES LANDSAT 8 EN LORETO, PERÚ
}

\author{
Juan J. PALACIOS ${ }^{1}$, Ricardo ZÁRATE ${ }^{1}$, Guiuseppe M. TORRES ${ }^{1}$, Nandy L. MACEDO², Aldo A. ALVA \\ Priscila Y. GONZALES ${ }^{4}$ \\ 1 Instituto de Investigaciones de la Amazonía Peruana. Programa de Investigación en Cambio Climático Desarrollo \\ Territorial y Ambiente (Proterra); Av. Quiñones km 2.5, San Juan Bautista, Maynas, Loreto, Perú. E-mail: \\ jpalacios@iiap.org.pe, rzarate@iiiap.org.pe \\ 2 Instituto de Investigaciones de la Amazonía Peruana. Programa de Investigación de Biodiversidad Amazónica; Av. \\ Quiñones km 2.5, San Juan Bautista, Maynas, Loreto, Perú. \\ 3 Universidad Nacional de la Amazonía Peruana; Jr. Pebas Quinta cuadra. Iquitos, Loreto, Perú. E-mail: \\ alvavelaaldo@gmail.com \\ 4 Servicios de Biodiversidad; Jr. Independencia 405, Maynas, Loreto, Perú.
}

\section{RESUMEN}

El objetivo del presente estudio es evaluar la reflectividad de los herbazales pantanosos y áreas deforestadas ubicadas en tierra firme, utilizando imágenes de satélite Landsat 8 y el programa ArcGIS 10. Se realizó la calibración radiométrica de las bandas espectrales 5 (infrarojo cercano), 4 (rojo) y 3 (verde), de las escenas 006-063 y 007-063. Se combinaron las bandas calibradas en rojo, verde y azul (RGB) para facilitar la visualización y digitalización de las áreas de muestreo. Se obtuvieron las reflectividades de los pixeles en las bandas rojo (R) e infrarojo cercana (IRC) por área de muestreo. Las reflectividades fueron analizadas a través de un ANOVA. Para interpretar la reflectividad se distribuyeron los valores en un plano IRC-R. Se observaron diferencias significativas en las reflectividades de los herbazales pantanosos y las áreas deforestadas. La reflectividad de los pixeles de las áreas deforestadas en la banda $\mathrm{R}$ tuvo un valor promedio de 0.0996, y en la banda IRC un promedio de 0.2534 ; mientras que en los herbazales pantanosos, en la banda R presentó un promedio de 0.0541 , y en la IRC una media de 0.2432 . La reflectividad de los herbazales pantanosos en el plano IRC-R muestra una mayor tendencia a valores de vegetación, y rara vez valores de suelo desnudo y agua. En las áreas deforestadas se observa exclusivamente una tendencia a valores de suelo desnudo. Se concluye que las imágenes Lansat 8 pueden ser utilizadas para diferenciar las áreas con herbazales pantanosos de aquellas áreas que están deforestadas.

PALABRAS CLAVE: Deforestación, Herbazal pantanoso, Landsat 8, Reflectividad.

\section{ASSESSING THE REFLECTIVITY OF MARSHY GRASSLANDS AND DEFORESTED AREAS USING LANDSAT 8 IMAGERY IN LORETO, PERU}

\begin{abstract}
The aim of this paper is to present the evaluation of the reflectivity of areas of marshy grasslands and deforestated areas in "terra firme" using Landsat 8 images and the ArcGIS 10 programme. Radiometric calibration of spectral bands 5 (near infrared), 4 (red) and 3 (green) of the scenes 006-063 and 007-063 was made. Bands calibrated were combined in RGB to facilitate viewing and digitizing of sampling areas. Reflectivities of the pixels in the red band (R) and near-infrared (NIR) were obtained for each sampling area. The reflectivities were analyzed through an ANOVA. The distribution of the reflectivity values in a NIR-R plane facilitated interpretation of the reflectivity. There were significant differences in the reflectivities of marshy grasslands and deforested areas. The reflectivity of pixels of the deforested areas, $\mathrm{R}$ had a mean of 0.0996 , and the NIR a mean of 0.2534 . On the other hand, in marshy grasslands R presented a mean of 0.0541 , and NIR a mean of 0.2432 . In the IRC-R plane, the reflectivity of marshy grasslands shows a greater tendency to values of vegetation, and a slight trend is observed to values of bare soil and water. In contrast, deforested areas show trends to values of only bare soil. The results of this study can be used to differentiate areas between marshy grasslands and deforested areas.
\end{abstract}

KEYWORDS: Deforestation, Landsat 8, Marshy grassland, Reflectivity. 


\section{INTRODUCCIÓN}

La Amazonía contiene la mayor diversidad de especies vegetales del planeta, albergando una gran clasificación de bosques. Según una de las clasificaciones más recientes se pueden distinguir dieciséis clases de cobertura vegetal, las cuales forman cuatro categorías de bosques densos, bosques abiertos, bosques inundables y vegetación no boscosa (Saatchi et al., 2008). Las "comunidades vegetales" con mayor expansión en los últimos años han sido los complejos de purmas, chacras y áreas sin vegetación, que corresponden a las áreas deforestadas (Josse et al., 2007).

Los herbazales son en particular un tipo de vegetación que se desarrolla sobre un sustrato pobre en oxígeno, de difícil drenaje por estar sobresaturado con agua. Se caracterizan por una estructura simple con presencia de herbáceas y arbustos que habitan en pequeñas áreas o cochas (Terborgh, 1990). La deforestación se define como la transformación del dosel de cobertura arbórea en un área desnuda o en otra comunidad vegetal dominada por hierbas, arbustos, árboles pioneros y cultivos agrícolas, debido a la actividades antrópicas que se desarrollan sobre ella o en el suelo que la sustenta (INRENA, 1996). Los herbazales pantanosos y las áreas deforestadas presentan muchas características en común, haciéndose presentes en las imágenes de satélite Landsat. Una de ellas es la reflectividad, entendida como la relación entre el flujo de energía incidente y su reflejo.

Entre los años 2000 y 2011, los Ministerios de Agricultura y Ambiente, elaboraron un mapa de la deforestación en la Amazonía peruana, con la finalidad de informar sobre la cobertura boscosa perdida y los lugares que habían sido más afectados por la misma. A mediados del año 2012 se procesaron las imágenes del mapa con CLASLITE 3.0 para la actualización del análisis de la cobertura forestal y la deforestación en la Amazonía peruana.

En la imagen de satélite Landsat 8, en una combinación en falso color RGB 543, los herbazales pantanosos son muy parecidos visualmente a las áreas deforestadas por las actividades humanas y podrían ser confundidas, lo cual puede traer como consecuencia una mala interpretación de las áreas analizadas.

El estudio de la cobertura vegetal y del suelo, mediante imágenes de satélite, se ha fundamentado en la respuesta radiométrica diferente que tienen el suelo y la vegetación (Melia et al., 1986). Espectralmente, el comportamiento de la vegetación en la banda del rojo (R) se debe a que la clorofila y otros elementos de las hojas absorben la mayor cantidad de la radiación solar, por lo que su reflectividad es mucho menor (Gates et al., 1965; Riaño et al., 2000). La alta reflectividad de la vegetación se debe a la estructura celular interna, lo que se aprecia en la banda del infrarojo cercano (Knipling, 1970; Riaño et al., 2000).

En la distribución de las reflectividades en un plano IRC-R, los puntos que determinan superficies desnudas se distribuyen a lo largo de una línea recta denominada "línea de suelo". A medida que la vegetación crece sobre un tipo de suelo determinado, disminuye la reflectividad en la banda $\mathrm{R}$ y aumenta en la banda IRC, por lo que el punto representativo de una cubierta vegetal va separándose de la línea del suelo en sentido ascendente y hacia la izquierda (Sobrino, 2000).

El presente trabajo tiene como objetivo evaluar la diferencia entre la reflectividad de los pixeles de las imágenes de satélite Landsat 8 en las bandas R e IRC de los herbazales pantanosos y las áreas deforestadas en un sector del departamento de Loreto, Perú.

\section{MATERIAL Y MÉTODOS}

\section{Área de estudio}

Comprende la cuenca baja de los ríos Nanay, Itaya y Tamshiyacu, afluentes del río Amazonas y la cuenca baja de los ríos Tigre, Chambira, Urituyacu y Nucuray, afluentes del río Marañón (Figura 1). Climáticamente toda la zona se caracteriza por ser cálida, tropical y húmeda durante todo el año. La precipitación anual es de 2000 a $3000 \mathrm{~mm} / \mathrm{año}$, distribuidos de tal forma que no presentan un período seco extenso y ningún mes con precipitación menor de $100 \mathrm{~mm}$, aunque los meses de junio a octubre son menos lluviosos que los meses de noviembre a mayo. La temperatura media anual oscila entre los $23^{\circ} \mathrm{C}$ a los $27^{\circ} \mathrm{C}$; la humedad relativa está cercana al $85 \%$ y es constante a lo largo del año. Las horas de sol promedio varían de 3 a 4 horas diarias durante febrero a abril y de 5.8 a 6.7 durante la estación de junio a setiembre (ONERN, 1982; Kvist \& Nebel, 2000; Conam, 2005; Lähteenoja, 2011; Paredes, 2012). La marcada variación estacional en la precipitación conduce a la formación de dos paisajes distintos en la Amazonia occidental: "tierra firme" (no inundables) y la planicie de inundación, que se inunda parcialmente durante la estación húmeda (Aniceto et al., 2014). El sector comprendido entre la cuenca baja del río Nanay y el río Tigre, corresponde a depósitos fluviales (terrazas inundables e islas), fluvio palustres recientes (pantanos herbáceos y arbustivos) y depósitos fluviales canalizados recientes y antiguos 
(complejos de orillares y meandros abandonados) del Holocéno; asimismo, depósitos palustres (terrazas no inundables con deficiencia de drenaje), depósitos aluviales antiguos (terrazas medias y altas arcillosas), las formaciones Iquitos Superior e Inferior (terrazas medias y altas arenosas) del Pleistoceno; las formaciones Nauta Superior e Inferior del Plioceno (colinas bajas y lomadas) y las formaciones El Porvenir y Pebas del Mioceno (terrazas inundables de ríos de origen Amazónicos, valles de quebradas y colinas bajas). El sector comprendido entre la margen derecha del río Tigre y la margen izquierda del río Nucuray, pertenece a las áreas más influenciadas por las inundaciones en la subcuenca Pastaza-Marañón, cuyos sedimentos mayormente oscuros de origen volcánico pertenecen al Holoceno o a lo mucho al Pleistoceno (Räsänen, 1993; Willink, 2005), en estas se encuentran principalmente depósitos fluviales recientes (terrazas inundables de los ríos Tigre, Chambira, Urituyacu y Nucuray), depósitos lacustres (meandros abandonados) inundados por aguas negras proveniente de los afluentes del Marañon y depósitos canalizados recientes y antiguos (complejo de orillares recientes y antiguos) inundados por las aguas del Marañón y en menor proporción depósitos palustres-lacustres antiguos (aguajales y pantanos de altura).

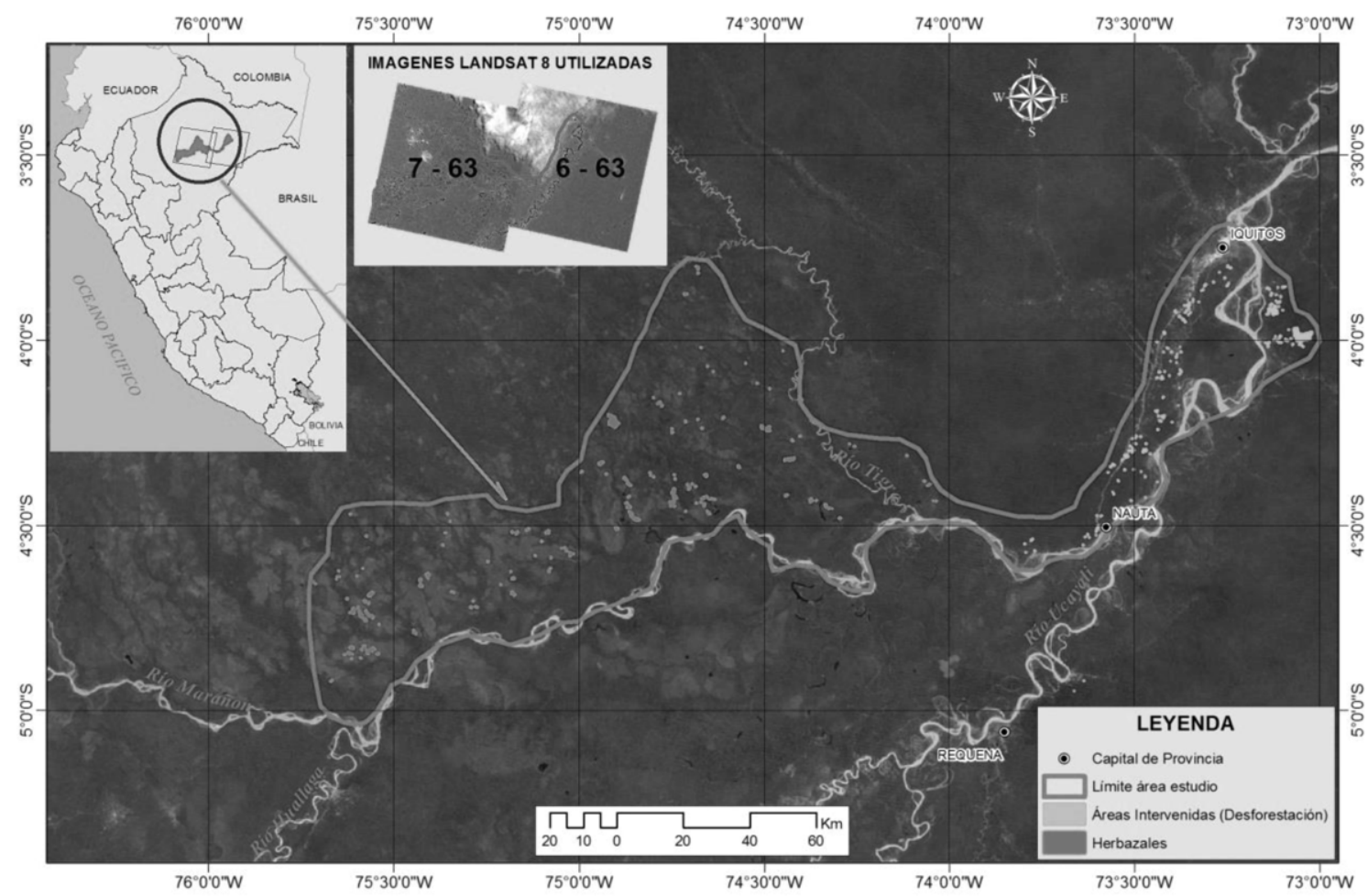

Figura 1. Mapa de ubicación del área de estudio. 


\section{Características de las comunidades vegetales estudiadas}

Herbazales pantanosos.- Son comunidades vegetales semiabiertas, con una matriz herbazal graminoide, asociadas en ocasiones con arbustos palustres (Josse et al., 2007) con mal drenaje del suelo, dominados por herbáceas arraigadas; los epífitos y los bejucos son escasos. La composición florística está representada por las especies: Pistia stratiotes, Centrosema brasilianum, Cyperus difformis, Eichhornia crassipes, Ludwigia sp. 1, Ludwigia sp. 2, Montrichardia arborescens, Panicum parvifolium. Triplaris peruviana, entre otras (Zárate, 2013).

Áreas deforestadas.- Comunidades vegetales de bosque abierto con presencia de plantas herbáceas y asociadas a especies pioneras en regeneración de bosques, toleran mucha intensidad de luz. Estas áreas fueron intervenidas por actividades antropogénicas como la roza, tumba y quema (MINAM, 2009) y abandonadas posteriormente, denominadas por la población local como "purmas". Está representada por las siguientes especies: Cecropia sciadophylla, Jacaranda copaia, Piper peltatum, Pueraria phaseoloides, Vismia amazonica, Phyllanthus stipulatus, Pourouma cecropiifolia, Saccharum officinarum (Zárate, 2013).

\section{Ubicación de las unidades de muestreo}

Para efectos de muestreo, el área de estudio fue dividida en dos sectores. En el primer sector, que comprende los herbazales pantanosos, se delimitaron 248 áreas de muestreo. El segundo sector, que comprende las áreas deforestadas, fue delimitada en 221 áreas de muestreo.

\section{Recolección de datos e información}

La recolección de datos e información satelital se realizó desde el servidor Earth Explorer del Servicio Geológico de los Estados Unidos USGS. Las imágenes escogidas para el presente estudio corresponden al satélite Landsat 8, sensor OLI, con resolución espacial $30 \mathrm{~m}$, resolución temporal 16 días, resolución radiométrica 8 bits, resolución espectral 11 bandas. El periodo de recogida de datos se llevó a cabo durante los meses de setiembre y octubre del 2013. Las escenas que recubren el área de estudio corresponden a la ubicación en path y row p006r063 y p007r063 (Figura 1).

\section{Corrección y procesamiento de imágenes}

El procesamiento de las imágenes en las bandas 5,4 y 3 , que corresponden al espectro visible (V) e infrarojo cercano (IRC), se inició con la corrección radiométrica de las bandas, lo cual permitió convertir los valores de pixeles de niveles digitales $\mathrm{ND}$ a valores de reflectividad aparente en el techo de la atmosfera TOA, con corrección para el ángulo solar cenital (De Asis \& Omasa, 2007; Hantson et al., 2011). Luego se realizó la fusión de bandas en la combinación RGB 543, para esto se utilizó el software ArcGIS 10.0.

\section{Delimitación de las áreas de muestreo}

Con las imágenes combinadas en RGB 543 y teniendo como referencia los puntos de verificación in situ, tomados y almacenados en un equipo GPS navegador, se digitalizó la capa vectorial de las áreas de muestra de herbazales pantanosos y áreas deforestadas, utilizando el software ArcGIS 10.0.

\section{Análisis de datos}

Se realizó una distribución de frecuencias para verificar sí los datos presentaban distribución normal. Se ejecutó el análisis de varianza (ANOVA) de un solo factor para determinar sí existían diferencias significativas entre la reflectividad de los pixeles de herbazales pantanosos y las áreas deforestadas (Ponzoni et al., 2002; Dytham, 2011). Los datos fueron analizados en el programa estadístico "R" disponible en http://www.rproject.org/.

\section{Distribución de reflectividades IRC-R}

Para interpretar los valores de reflectividad, se extrajeron los valores de los pixeles correspondientes a los herbazales pantanosos y las áreas deforestadas, los cuales fueron distribuidos independientemente en un plano IRC-R (Gilabert et al., 1997).

\section{RESULTADOS Y DISCUSIÓN}

La reflectividad de los pixeles en las áreas deforestadas en la banda del Rojo (R) varió de 0.0515 a 0.1956 , con una media de 0.0996 $( \pm 0.0118)$, desviación estándar de 0.0118 y el coeficiente de variación de $11.85 \%$; en la banda del Infra Rojo Cercano (IRC) varió de 0.15911 a 0.4481 , con una media de 0.2534, desviación estándar de 0.0315 y coeficiente de variación de $12.43 \%$. Mientras que en herbazales pantanosos, en la banda $\mathrm{R}$, varió de 0.0387 a 0.2891 , con una media de 0.0541 , desviación estándar de 0.0116 y el coeficiente de variación de $21.44 \%$; en la banda IRC varió de 0.1023 a 04672 , con una media de 0.2432 , desviación estándar de 0.0301 y coeficiente de variación de $12.38 \%$. 
La reflectividad de los herbazales y áreas deforestadas, de acuerdo al ANOVA, difiere tanto en la banda R como en la banda IRC. Esto indica que los valores de reflectividad son diferentes estadísticamente, lo que permitirá reforzar la interpretación visual de los herbazales pantanosos y áreas deforestadas utilizando imágenes de satélite Landsat 8, combinadas en RGB, ya sea en color verdadero o falso. En la Figura 2, se observa que la distribución de las reflectividades para herbazales pantanosos en el plano IRC-R, muestra una mayor concentración en valores correspondientes a la vegetación, sin embargo, se observa también que formando el triángulo de reflectividades se encuentra una ligera tendencia a valores de suelo desnudo y agua (Gilabert et al., 1997; Vercher et al., 2002), mientras que en las áreas deforestadas se observa una tendencia a valores de suelo desnudo, no formándose el triángulo de reflectividades (Figura 3).

Los resultados de este estudio pueden ser utilizados para diferenciar áreas con herbazales pantanosos de las áreas deforestadas, así como también en la elaboración de estudios de descripción de cobertura vegetal y cambio de cobertura y uso del suelo.

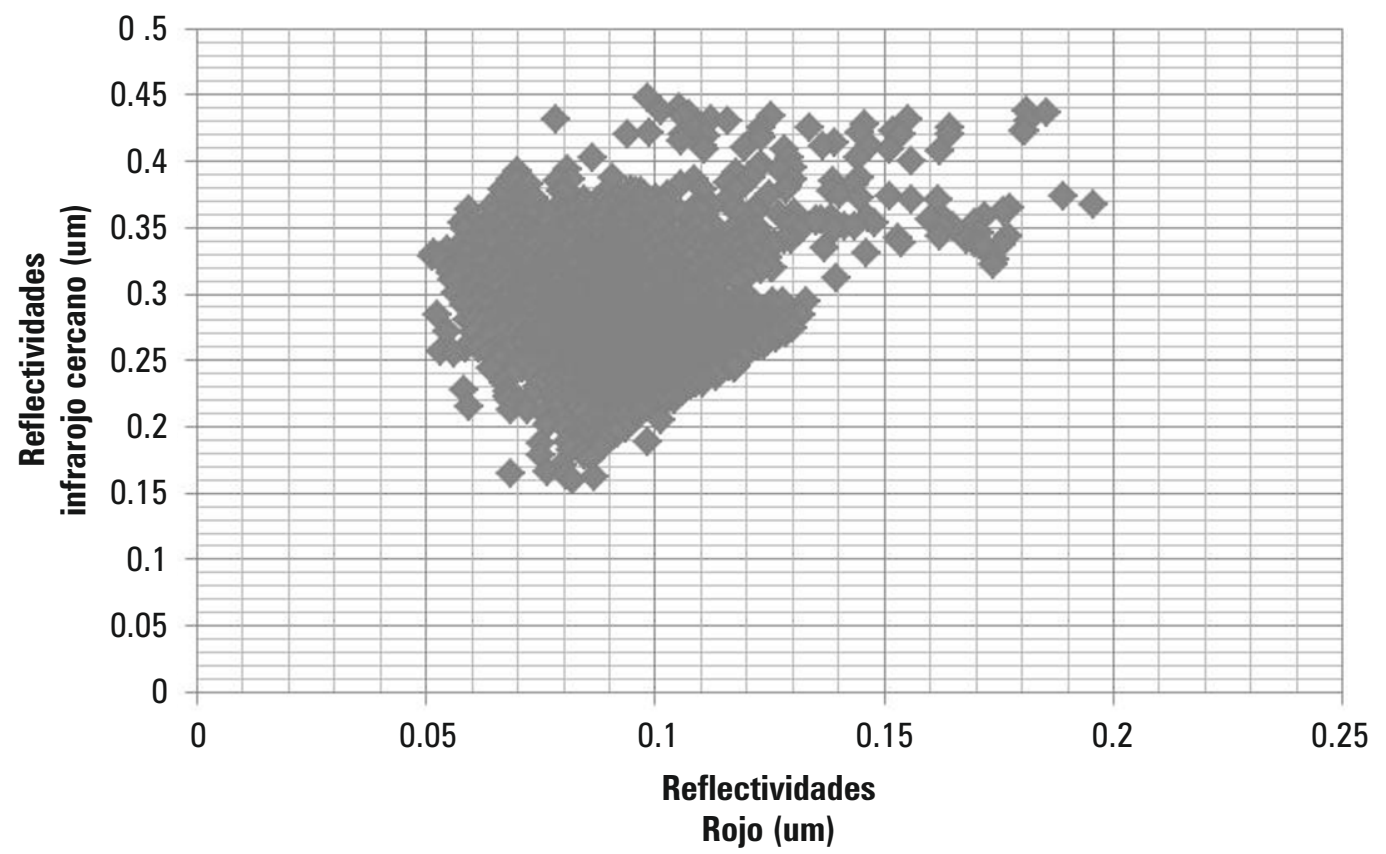

Figura 2. Plano de distribución IRC-R para las áreas muestra de deforestación, imagen LandSat 8 , setiembre 2013. 


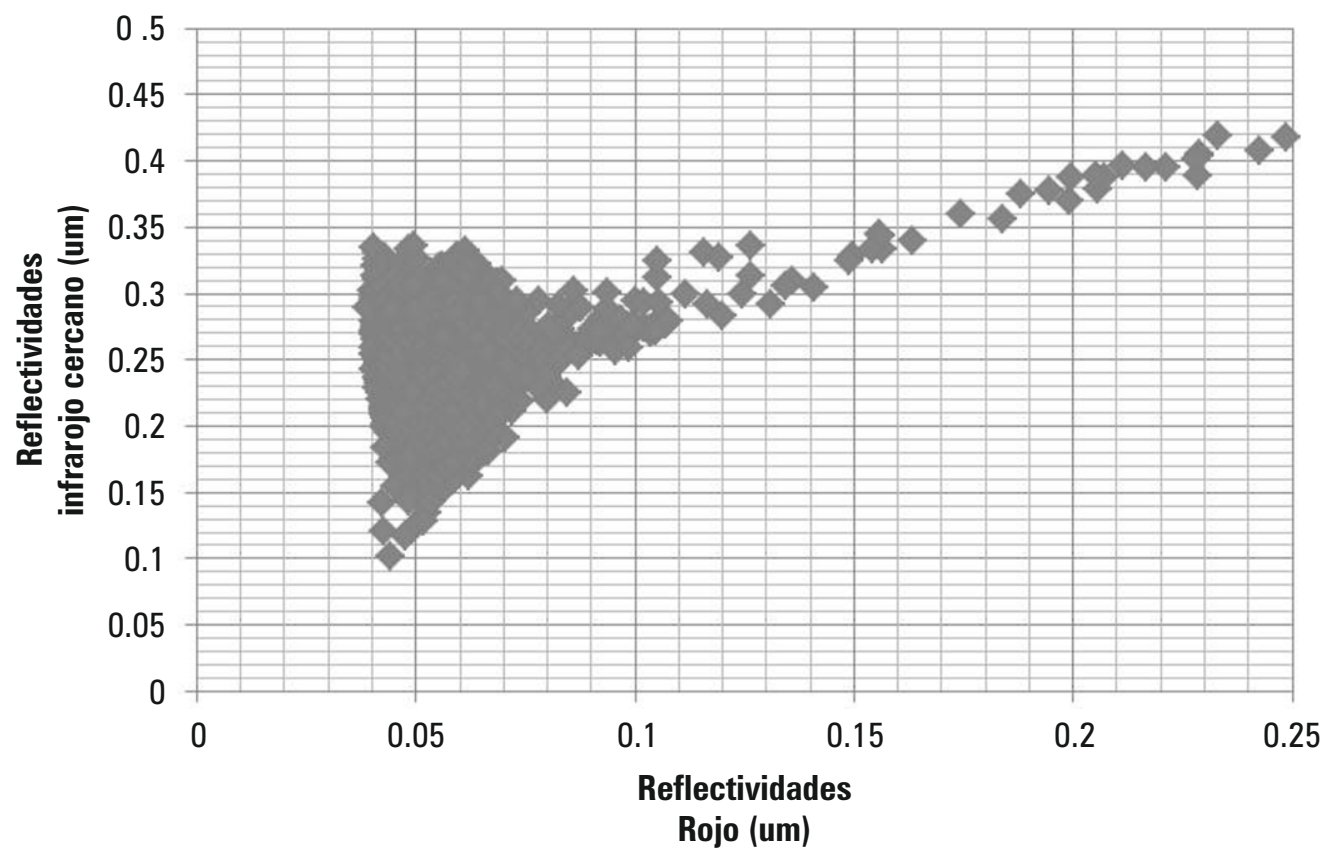

Figura 3. Plano de distribución IRC-R para las áreas muestra de herbazales pantanosos, imagen LandSat 8, setiembre 2013.

Tabla 1. Análisis de varianza de un factor banda del rojo (R).

\begin{tabular}{|c|c|c|c|c|c|c|}
\hline \multicolumn{7}{|l|}{ RESUMEN } \\
\hline Grupos & Cuenta & Suma & Promedio & Varianza & & \\
\hline 0.0387382 & 13878 & 750.240957 & 0.05405973 & 0.00013494 & & \\
\hline 0.0515197 & 9095 & 905.812299 & 0.09959454 & 0.00013975 & & \\
\hline \multicolumn{7}{|c|}{ ANÁLISIS DE VARIANZA } \\
\hline $\begin{array}{c}\text { Origen de las } \\
\text { variaciones }\end{array}$ & $\begin{array}{l}\text { Suma de } \\
\text { cuadrados }\end{array}$ & $\begin{array}{c}\text { Grados de } \\
\text { libertad }\end{array}$ & $\begin{array}{l}\text { Promedio de } \\
\text { los cuadrados }\end{array}$ & $\mathrm{F}$ & Probabilidad & $\begin{array}{c}\text { Valor crítico } \\
\text { para } \mathrm{F}\end{array}$ \\
\hline Entre grupos & 11.3919694 & 1 & 11.3919694 & 83248.4824 & 0 & 3.84186367 \\
\hline Dentro de los grupos & 3.14341982 & 22971 & 0.00013684 & & & \\
\hline Total & 14.5353892 & 22972 & & & & \\
\hline
\end{tabular}


Tabla 2. Análisis de varianza de un factor banda del infrarojo cercano (IRC)

\begin{tabular}{|c|c|c|c|c|c|c|}
\hline \multicolumn{7}{|l|}{ RESUMEN } \\
\hline Grupos & Cuenta & Suma & Promedio & Varianza & & \\
\hline 0.15911 & 9095 & 2304.85704 & 0.25342024 & 0.00099204 & & \\
\hline 0.102298 & 13878 & 3375.66026 & 0.24323824 & 0.00090452 & & \\
\hline \multicolumn{7}{|c|}{ ANÁLISIS DE VARIANZA } \\
\hline $\begin{array}{c}\text { Origen de las } \\
\text { variaciones }\end{array}$ & $\begin{array}{l}\text { Suma de } \\
\text { cuadrados }\end{array}$ & $\begin{array}{l}\text { Grados de } \\
\text { libertad }\end{array}$ & $\begin{array}{l}\text { Promedio de } \\
\text { los cuadrados }\end{array}$ & $\mathrm{F}$ & Probabilidad & $\begin{array}{l}\text { Valor crítico } \\
\text { para } \mathrm{F}\end{array}$ \\
\hline Entre grupos & 0.56961037 & 1 & 0.56961037 & 606.507403 & $3.332 \mathrm{E}-132$ & 3.84186367 \\
\hline Dentro de los grupos & 21.5735535 & 22971 & 0.00093916 & & & \\
\hline Total & 22.1431639 & 22972 & & & & \\
\hline
\end{tabular}

\section{AGRADECIMIENTOS}

Al Servicio Geológico de los Estados Unidos (USGS) por la disponibilidad de las imágenes de satélite. A Pedro Pérez Peña por la orientación en el análisis estadístico.

\section{REFERENCIAS BIBLIOGRÁFICAS}

Aniceto, K.; Moreira-Turcq, P.; Cordeiro, R.; Fraizy, P.; Quintana, I.; Turcq, B. 2014. Holocene paleohydrology of Quistococha Lake (Peru) in the upper Amazon Basin: Influence on carbon accumulation, Palaeogeogr. Palaeoclimatol. Palaeoecol, http://dx.doi.org/10.1016/j.palaeo. Acceso: 2014.08.018.

BIODAMAZ. 2004. Diversidad de Vegetación de la Amazonía Peruana expresada en un mosaico de imágenes de satélite. Documento Técnico $\mathrm{N}^{\circ} 12$. Serie BIODAMAZ-IIAP. Iquitos, Perú. 68 pp.

CONAM. 2005. Indicadores Ambientales Loreto. Serie Indicadores Ambientales $\mathrm{N}^{\circ} 07.60 \mathrm{pp}$.

De Asis, A.M.; Omasa, K. 2007. Estimation of vegetation parameter for modeling soil erosion using linear Spectral Mixture Analysis of Landsat ETM data. ISPRS-J Photogramm Remote Sens 62(3): 309-324.

Dytham, Calvin. 2011. Choosing and using statistics: a biologist's guide/by Calvin Dytham. -3 rd ed. 320 pp.

Gates, D. M.; Keegan, H. J.; Schleter, J. C.; Weidner, V. R. 1965. Spectral propierties of plants. Applied Optics. 4: 11-20.
Gilabert, M. A.; González-Piqueras, J.; GarcíaHaro, J. 1997. "Acerca de los índices de vegetación", Revista de Teledetección, vol. (8): 35-45.

Hantson, S.; Chuvieco, E.; Pons, X.; Domingo, C.; Cea, C.; Moré, G.; Cristobal, J.; Peces, J. J.; Tejeiro, J. A. 2000. Cadena de preprocesamiento estándar para las imágenes Landsat del Plan Nacional de Teledetección. Revista de Teledetección, vol. 36: 51-61.

Instituto Nacional de Recursos Naturales (INRENA) (1996b). Monitoreo de la Deforestación en la Amazonía Peruana. Lima: Dirección General de Medio ambiente Rural, 35 $\mathrm{pp}$.

Josse, C.; Navarro, G.; Encarnación, F.; Tovar A.; Comer, P.; Ferreira, W.; Rodríguez, F.; Saito, J.; Sanjurjo, J.; Dyson, J.; Rubin de Celis E.; Zárate, R.; Chang, J.; Ahuite, M.; Vargas, C.; Paredes, F.; Castro, W.; Maco J.; Reátegui, F. 2007. Sistemas Ecológicos de la Cuenca Amazónica de Perú y Bolivia. Clasificación y mapeo. NatureServe, Virginia, USA. 94 pp.

Knipling, E. B. 1970. Physical and Physiological basis for the reflectance of visible and nearinfrared radiation from vegetation. Remote Sensing Environ. 155-159.

Kvist, L. P.; Nebel, G. 2000. Bosque de la llanura aluvial del Perú: ecosistemas, habitantes y uso de los recursos. Folia Amazónica VOL. 10 (1-2): 555. 
Lähteenoja, Outi. 2011. Carbon Dynamics and Ecosystem Diversity of Amazonian Peatlands. Turun Yliopiston Julkaisuja. Annales Universitatis Turkuensis. Sarja - Ser. All Osa Tom. 264. Biologica- Geographica - Geologica. $38 \mathrm{pp}$.

Melia, J. 1986. "Introducción a las técnicas de teledetección y sus aplicaciones agronómicas", Facultad de Ciencias Físicas, Dpto. de Termología, Universidad de Valencia. 409 pp.

MINAM. 2009. Mapa de deforestación de la Amazonía Peruana-2000. Lima-Perú. 17 pp.

ONERN, 1982. Estudio detallado de suelos de la zona del río Manití- Iquitos. Vol. I. 46 pp.

Paredes, M. 2012. Clima. Documento Temático. Micro Zonificación Ecológica y Económica para el Desarrollo Sostenible del Área de Influencia de la Carretera Iquitos-Nauta, convenio entre el IIAP y DEVIDA. Iquitos - Perú. 88 pp.

Räsänen, M. 1993. La geohistoria y geología de la Amazonía Peruana. In: Kalliola, R.; M. Puhakka and W. Danjoy (eds.). Amazonía Peruana. Vegetación húmeda tropical en el llano subandino. PAUT \& ONERN, Jyväskylä. p.4367.

Riaño, D.; Vaughan, P.; Chuvieco, E. 2000. Bases para estimar el contenido de humedad en plantas mediterráneas a partir de la teledetección. Mediterránea: Serie de Estudios Biológicos, 2(17): 27-34.

Saatchi, S., Buermann, W., Mori, S. and ter Steege, H. (2008) Modeling distribution of Amazonian tree species and diversity using remote sensing measurements. Remote Sensing of Environment, 112 (5): 2000-2017.
Sobrino, J. A. 2000. Teledetección. AECI, Valencia. 467pp.

Terborgh, 1990. Línea Base Biológica - EIAS de la prospección sísmica. Capítulo 4.2.190 pp.

Vercher, A.; Gilabert, M. A.; Camacho de Coca, F.; Meliá, J. 2002. Influencia del ángulo cenital de iluminación en los índices de vegetación. Revista de Teledetección, 18: 75-89.

Willink, P.W., B. Chernoff, and W.A. Palacios. 2005. Geology, geomorphology, and climate of the Pastaza River basin. In A biological assessment of the aquatic ecosystems of the Pastaza River basin, Ecuador and Perú. Pp. 30-32. In A Rapid Biological Assessment of the Aquatic Ecosystems of the Pastaza River Basin, Ecuador and Perú. RAP. Willink, P.W.; Chernoff, B.; McCullough, J. (eds.). Bulletin of Biological Assessment 33. Conservation International, Washington, DC.

Yanggen, D. 1999. Deforestación en la Selva Peruana: Un análisis del impacto de los diversos productos agropecuarios y tecnologías de producción. Perspectivas del Medio Ambiente en la Amazonía. Universidad del Pacífico. $608 \mathrm{pp}$.

Zárate, R.; Mori, T. y Maco, J. 2013. Estructura, Composición y Diversidad Florística de las Comunidades Vegetales del ámbito de la Carretera Iquitos-Nauta, Loreto, Perú. Folia Amazónica, 22 (1-2): 77-89.

Recibido: 23 de febrero del 2015

Aceptado para publicación: 15 de abril del 2015 